\title{
Dernières connaissances scientifiques de risques cardiovasculaires dus aux anti- inflammatoires non stéroïdiens et leurs conséquences pour les textes d'information sur les médicaments
}

Les anti-inflammatoires non stéroïdiens (AINS) sont principalement utilisés pour le traitement symptomatique des formes inflammatoires et dégénératives des rhumatismes de types ostéoarthrose et polyarthrite rhumatoïde. L'efficacité de tous les AINS actuellement commercialisés en Suisse a été établie dans les indications autorisées par des essais cliniques randomisés contrôlés.

Toutefois, un nouveau risque a été établi à l'automne dernier concernant deux AINS dits sélectifs de la COX-2 (le rofécoxib et le célécoxib). Des études contrôlées de grande ampleur contre placebo, sur une longue période de traitement (3 ans), portant sur une nouvelle application de ces médicaments (prévention des tumeurs du côlon), ont en effet révélé un accroissement $\mathrm{du}$ risque de complications thrombotiques (par rapport au placebo) lors de la prise de rofécoxib $\left(\right.$ Vioxx $\left.^{\circledR}\right)$ et de célécoxib (Celebrex $\left.{ }^{\circledR}\right)$. Ces résultats, nouveaux, ont conduit au retrait de Vioxx ${ }^{\circledR}$ et à une limitation d'emploi de Celebrex ${ }^{\circledR}$. De plus, l'autorisation de Bextra ${ }^{\circledR}$ (valdécoxib/parécoxib) a été suspendue au printemps 2005 pour cause d'aggravation du risque d'effets secondaires cardiovasculaires et allergiques.

Swissmedic a informé à plusieurs reprises les spécialistes et la population des nouvelles données en matière de risques découlant de l'utilisation de AINS sélectifs de la COX-2 et de leurs limitations d'emploi [1-5].

Deux nouvelles études ont été publiées le 11 juin 2005 dans le British Medical Journal sur le risque de maladies cardiovasculaires également sous traitement par AINS non sélectifs [6, 7]. Les résultats indiquent que l'utilisation des AINS étudiés - qu'ils soient sélectifs de la COX-2 ou non - accroît le risque cardiovasculaire. Cependant, dans la mesure où il s'agit en l'occurrence d'études épidémiologiques rétrospectives fondées sur des données existantes («étude castémoins au sein d'une cohorte» ou «nested case control study» et «étude de cohorte rétrospec- tive» ou «retrospective cohort study»), ces résultats ne peuvent être considérés comme probants. Ceci est d'ailleurs mentionné à la fois par les auteurs des études et dans l'éditorial qui les accompagne. Dans le cadre de l'examen en cours sur le risque cardiovasculaire posé par les AINS, Swissmedic tiendra aussi compte de la publication de ces dernières données scientifiques en s'appuyant sur les études cliniques contrôlées.

Dans son information adressée aux professionnels de la santé sur les risques des AINS, Swissmedic avait déjà indiqué en octobre 2004 qu'aucune corrélation claire n'avait été prouvée entre la sélectivité des AINS vis-à-vis de la COX2 et le risque cardiovasculaire [1].

Il reste que l'actuelle évidence scientifique en la matière ne permet pas d'exclure la présence de ce risque pour les AINS non sélectifs. Il faudrait en effet, afin de démontrer son absence ou sa réalité, que des études contrôlées contre placebo, de taille comparable et à long terme, aient été réalisées individuellement sur tous les AINS, comme cela a été le cas pour le rofécoxib et le célécoxib. Or, aucune étude de ce type portant sur d'anciens AINS n'a pour l'instant été soumise à Swissmedic. C'est la raison pour laquelle l'institut a fait savoir dans un communiqué paru en avril 2005 que les AINS non sélectifs feraient eux aussi l'objet d'un examen afin d'évaluer les risques cardiovasculaires qui leur sont liés [5].

\section{Recommandations actuelles de Swissmedic concernant les AINS}

1. Comme pour tout autre médicament, il convient systématiquement, avant de prescrire un AINS (indépendamment de sa sélectivité vis-à-vis de la COX-2), de peser soigneusement le rapport entre le bénéfice que l'on peut en attendre et le risque potentiel d'effets secondaires. Outre les possibles complications cardiovasculaires, il faut ainsi 
également prendre en considération les risques de complications gastro-intestinales, rénales, hépatiques et cutanées.

2. Sur la base des évidences scientifiques actuelles, le médecin, lors de la prescription d'AINS non sélectifs, devra tenir compte des contreindications et des mesures de précautions suivantes:

\section{Contre-indications}

- Hypersensibilité par rapport au principe actif ou à l'un des excipients conformément à la composition;

- anamnèse de bronchospasme, d'urticaire ou de symptômes semblables à une manifestation allergique après prise d'acide acétylsalicylique ou d'autres anti-inflammatoires non stéroïdiens;

- troisième trimestre de la grossesse (cf. «Grossesse, allaitement»);

- ulcères gastriques et/ou duodénaux actifs ou saignements gastro-intestinaux;

- maladies intestinales inflammatoires (p.ex. maladie de Crohn, colite ulcéreuse);

- troubles sévères de la fonction hépatique (cirrhose hépatique et ascite);

- insuffisance rénale sévère (clairance de la créatinine $<30 \mathrm{ml} / \mathrm{min}$.);

- insuffisance cardiaque sévère (III-IV);

- traitement de douleurs postopératoires après un pontage coronaire (ou après l'utilisation d'une machine cœur-poumons).

Pour d'éventuelles contre-indications supplémentaires propres à un AINS en particulier, se reporter à la publication actuellement publiée de l'information professionnelle concernant le médicament.

\section{Mesures de précaution}

Des perforations, des ulcères et des saignements gastro-intestinaux peuvent être observés chez des patients traités avec des anti-inflammatoires non stéroïdiens (AINS), sélectifs de la COX-2 ou non. Ces effets indésirables peuvent survenir à tout moment, sans signes annonciateurs ni antécédents connus. Pour réduire ce risque, il convient donc d'administrer la dose efficace la plus faible, pendant une durée de traitement aussi courte que possible.

Des études contrôlées contre placebo ont mis en évidence pour certains inhibiteurs sélectifs de la COX-2 une augmentation du risque de complications cardiovasculaires et cérébrovasculaires thrombotiques. Mais on ignore pour l'heure si ce risque est en corrélation directe avec la sélectivité COX-1/COX-2 des AINS. Pour les substances actives pour lesquelles aucune donnée issue d'études cliniques comparables n'est actuellement disponible à la posologie maximale et dans le cadre d'un traitement à long terme, une augmentation analogue du risque ne peut être exclue. Par conséquent, jusqu'à ce que de telles données soient disponibles, ces substances actives ne devraient être administrées qu'après évaluation minutieuse du rapport bénéfice/risque en cas de cardiopathie ischémique avérée, de maladies cérébrovasculaires, d'artériopathies obstructives périphériques ou aux patients présentant d'importants facteurs de risque cardiovasculaires (p. ex. hypertension, hyperlipidémie, diabète sucré, tabagisme). Eu égard également à ce risque, il convient d'administrer la dose efficace la plus faible, pendant une durée de traitement aussi courte que possible.

Les effets rénaux des AINS incluent la rétention hydrique avec oedèmes et/ou hypertension artérielle. Chez les patients présentant des troubles de la fonction cardiaque et d'autres états qui les prédisposent à la rétention hydrique, un AINS ne devrait donc être utilisé qu'avec prudence. Celle-ci est également de rigueur chez les patients qui prennent simultanément des diurétiques ou des inhibiteurs de l'enzyme de conversion de l'angiotensine et en cas de risque élevé d'hypovolémie.

Pour d'éventuelles mises en garde ou précautions supplémentaires propres à un AINS en particulier, se reporter à la publication actuelle de l'information professionnelle concernant le médicament.

- Les patients ne devraient pas interrompre le traitement qui leur a été prescrit sans avoir au préalable consulté leur médecin.

- Au vu des données scientifiques actuellement disponibles, la conclusion publiée par Swissmedic en octobre 2004 reste pertinente: Le rapport bénéfice/risque global des AINS reste positif pour chaque indication autorisée, pour autant que les contre-indications, mesures de précaution et mises en garde soient prises en compte.

- Pour les AINS non soumis à ordonnance, qui sont utilisés à faibles doses au titre d'analgésiques, il convient de respecter strictement la posologie recommandée et la durée de traitement maximale (3 jours). 


\section{Références}

Pour consulter les références 1 à 5 voir www. swissmedic.ch

1 Sécurité d'emploi des anti-inflammatoires: état actuel des connaissances (20.10.2004).

2 Prise de position de Swissmedic sur la suspension prématurée d'un essai clinique avec Celebrex (20.12.2004)

3 Interruption d'une étude clinique avec le naproxène aux Etats-Unis (21.12.2004).

4 Evaluation des risques liés aux anti-inflammatoires inhibiteurs de la COX-2: derniers développements dans l'UE et aux Etats-Unis (19.2.2005)
5 Suspension immédiate de la distribution de Bextra ${ }^{\circledR}$ comprimés filmés (7.4.2005).

6 Hippisley-Cox J, Coupland C. Risk of myocardial infarction in patients taking cyclo-oxygenase-2 inhibitors or conventional non-steroidal antiinflammatory drugs: population based nested case-control analysis. BMJ 2005;330:1366.

7 Hudson M, Richard H, Pilote L. Differences in outcomes of patients with congestive heart failure prescribed celecoxib, rofecoxib, or non-steroidal anti-inflammatory drugs: population based study. BMJ 2005;330:1370. 